Gut, 1968, 9, 7-16

\title{
Crohn's disease of the colon and its distinction from diverticulitis
}

\author{
G. T. SCHMIDT, J. E. LENNARD-JONES, B. C. MORSON, AND \\ A. C. YOUNG
}

From St. Mark's Hospital, London

Crohn's disease may affect segments of the colon (Brooke, 1959; Lindner, Marshak, Wolf, and Janowitz, 1963; Lockhart-Mummery and Morson, $1960,1964)$ where diverticula are a common finding in the middle-aged and elderly (Dearlove, 1954; Pemberton, Black, and Maino, 1947). It would thus be surprising if Crohn's disease did not sometimes occur in the region of colonic diverticula and the object of this paper is to describe 26 such patients.

Some of these patients presented little diagnostic difficulty. Others, with colonic diverticula, presented as 'diverticulitis' but the inflammation of the colon was subsequently shown to be due to Crohn's disease. Patients have also been seen who were diagnosed as diverticulitis but were later found to have Crohn's disease without any diverticula being present.

\section{NOMENCLATURE}

In this paper the term 'diverticulosis' is used to describe the presence of colonic diverticula as shown radiologically and/or pathologically. The term 'diverticular disease' is used to describe that condition in which there is an abnormality of muscle of the sigmoid colon with or without diverticula (Morson, 1963; Williams, 1963; Arfwidsson, 1964). 'Diverticulitis' is used here only when inflammation has occurred as a complication of diverticulosis. The use of the expression 'Crohn's disease' has been described in previous papers (Lockhart-Mummery and Morson, 1960, 1964). It is synonymous with the terms 'regional enteritis' (Crohn and Yarnis, 1958) and 'granulomatous colitis' (Lindner et al., 1963).

\section{CLINICAL MATERIAL}

The records of 137 patients with Crohn's disease of the colon have been reviewed and 26 were found in which both diverticula and Crohn's disease were present. The cases have been analysed and divided into two groups.
GROUP I: DIAGNOSIS MADE ON A RESECTED SPECIMEN Fourteen patients have been seen with Crohn's disease and diverticula of the sigmoid colon in whom the diagnosis was established by pathological examination of a resected specimen. Nine of these 14 patients were diagnosed clinically as diverticulitis, four as Crohn's disease, and one as ulcerative colitis. Crohn's disease was diagnosed in the presence of colonic diverticula in 10 of the 14 specimens when first examined but in the other four only after review of the pathology following the development of characteristic postoperative complications such as anal or entero cutaneous fistulae.

Case 1 A 69-year-old man complained of intermittent rectal bleeding for four years, left-sided lower abdominal pain, and recent diarrhoea. He was found to have an unhealed anal fistula which had been present for two years: sigmoidoscopy was normal to $15 \mathrm{~cm}$. Radiographs showed colonic diverticula and a diagnosis of diverticulitis was made.

At laparotomy a large mass, presumed to be diverticulitis, was found in the sigmoid region and diverticula extending to the proximal transverse colon were also noted. The mass was resected and on pathological examination was thought to show diverticulitis.

Within 12 months the patient returned with a discharging sinus in the left iliac fossa, continued rectal bleeding, and a chronic anal fissure. At laparotomy an entero-colic fistula was closed, an abscess around the anastomosis was drained, and a proximal colostomy performed. Following this he made an uneventful recovery but died from an unrelated cause some months later.

In view of the breakdown of the anastomosis and development of a fistula after the first operation the histology of the resected colon (Fig. 1) was reviewed. Mucosal ulceration (Fig. 2) with transmural inflammation and fissuring (Fig. 3) and foci of epithelioid and giant cells were found and an amended diagnosis of Crohn's disease of the sigmoid colon associated with diverticula was made. Examination of a biopsy specimen from the anal fistula taken 15 months before his referral to St. Mark's also revealed foci of sarcoid tissue consistent with a diagnosis of Crohn's disease. 


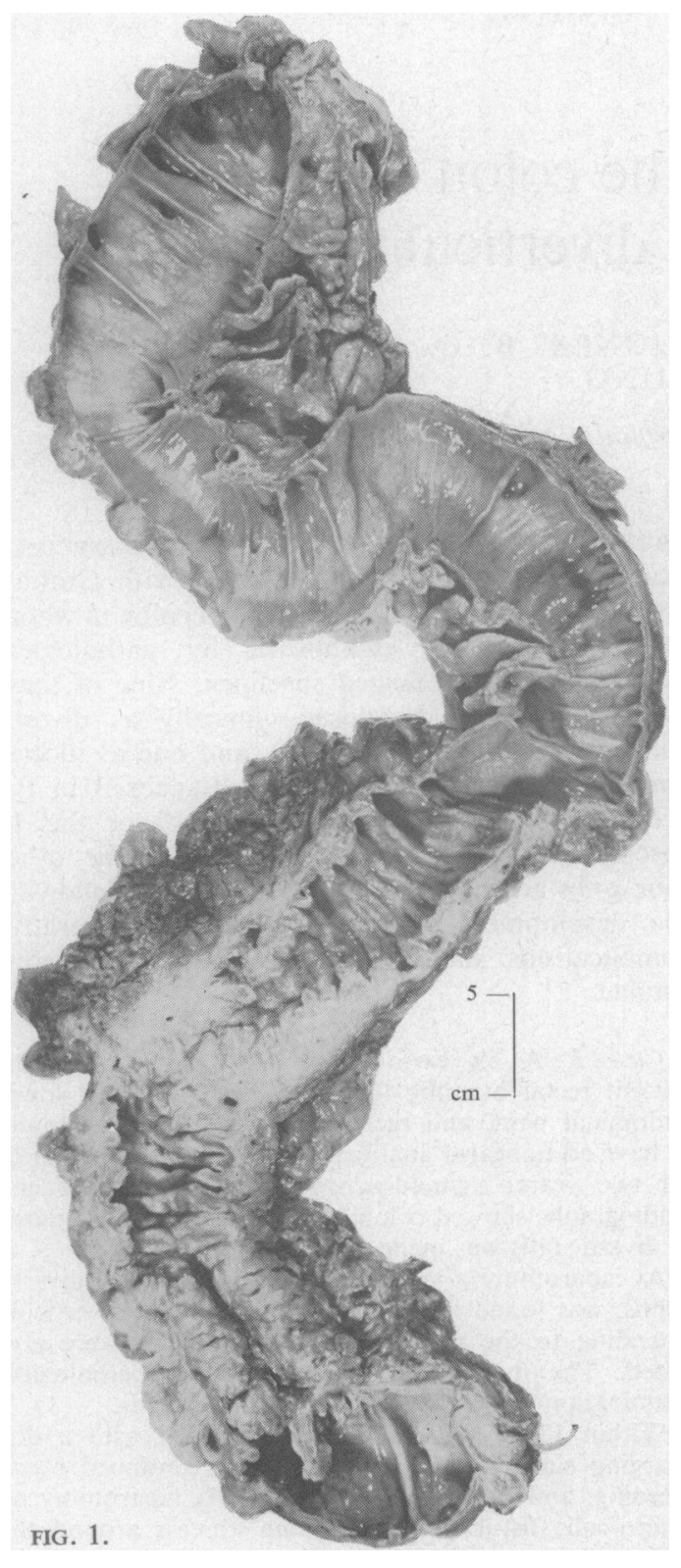

FIG. 1. Case 1: surgical specimen of descending and sigmoid colon. There is muscular thickening and corrugation of the bowel wall with mucosal redundancy in the sigmoid colon and extensive diverticulosis throughout the specimen. In the sigmoid there is also fibrous thickening of the bowel wall with stenosis of the lumen.

FIG. 2. Case 1: close-up of descending colon from Fig. 1 showing patchy irregular ulceration around the orifices of diverticula.

FIG. 3. Case 1: close-up of sigmoid part of Fig. 1 showing fibromuscular thickening of the bowel wall extending into the pericolic fat. The mucosa is granular and nodular and fissuring with intramural abscess formation is present.

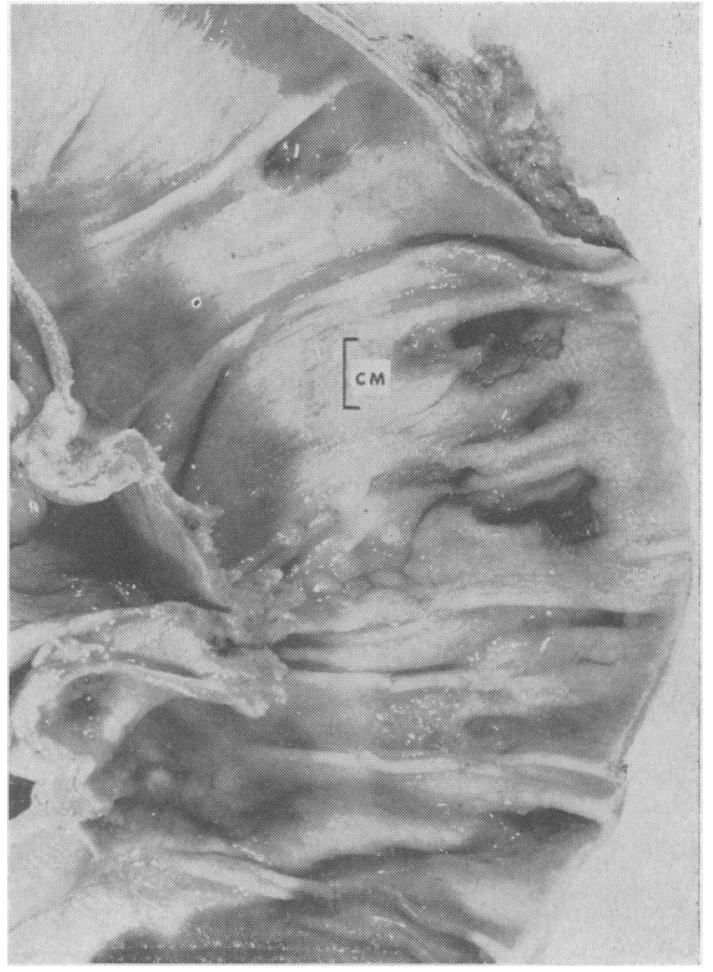

FIG. 2 .

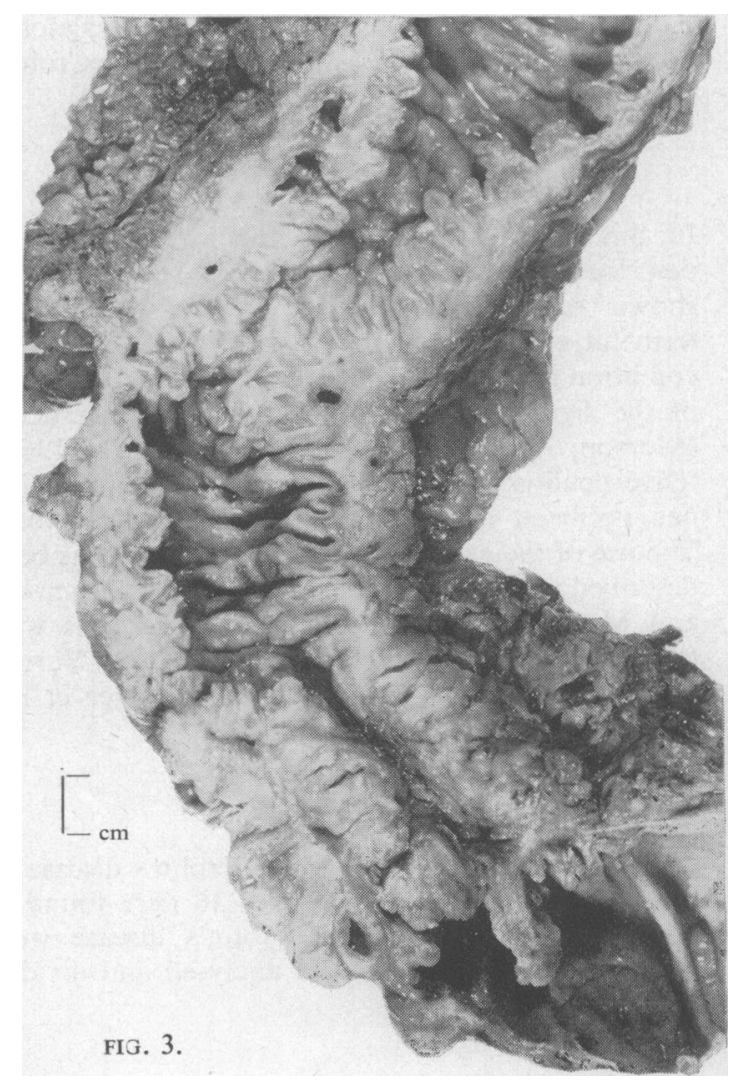


Case 2 A 55-year-old man was referred to St. Mark's Hospital suffering from an anal fistula which had been causing pain and discharge for two months. Bleeding with defaecation had occurred at the onset of his symptoms but he did not complain of any change of bowel habit or of any significant abdominal pain. Barium enema (Fig. 4) showed the asymmetrical fold pattern and narrowing of diverticular disease with a few diverticula and some spiky folds thought to be a localized peridiverticular abscess. The anal fistula was laid open and subsequently healed satisfactorily.

Five months later he returned complaining of abdominal pain, of alternating constipation and diarrhoea, and of loss of 1 stone in weight over six months. Sigmoidoscopy was normal and the anal fistula appeared to have healed completely.

The pain persisted and was attributed to diverticulitis. At operation a segment of inflamed sigmoid colon which was adherent to the adjacent bowel and ureter was resected. The operative diagnosis was diverticulitis but histological examination of the specimen showed Crohn's disease and diverticulosis. $\mathrm{He}$ made an uneventful recovery and barium examination of the small bowel revealed no changes of Crohn's disease. Review of the original enema films suggested that the spiky pattern seen was due to Crohn's disease rather than a peridiverticular abseess.

Case 3 A woman of 72 years was admitted complaining of diarrhoea, anorexia, and loss of weight over a period of 18 months. On examination a mass was felt in the left iliac fossa, and inspection of the anus revealed large oedematous skin tags. Sigmoidoscopy showed pus, blood, and mucus in the rectum with hyperaemia and oedema of the mucosa. Barium enema (Fig. 5) showed narrowing of the lumen of the descending and sigmoid colon associated with multiple diverticula and a diagnosis of diverticulitis with paracolic abscess was made.

At operation most of the colon was found to be in-

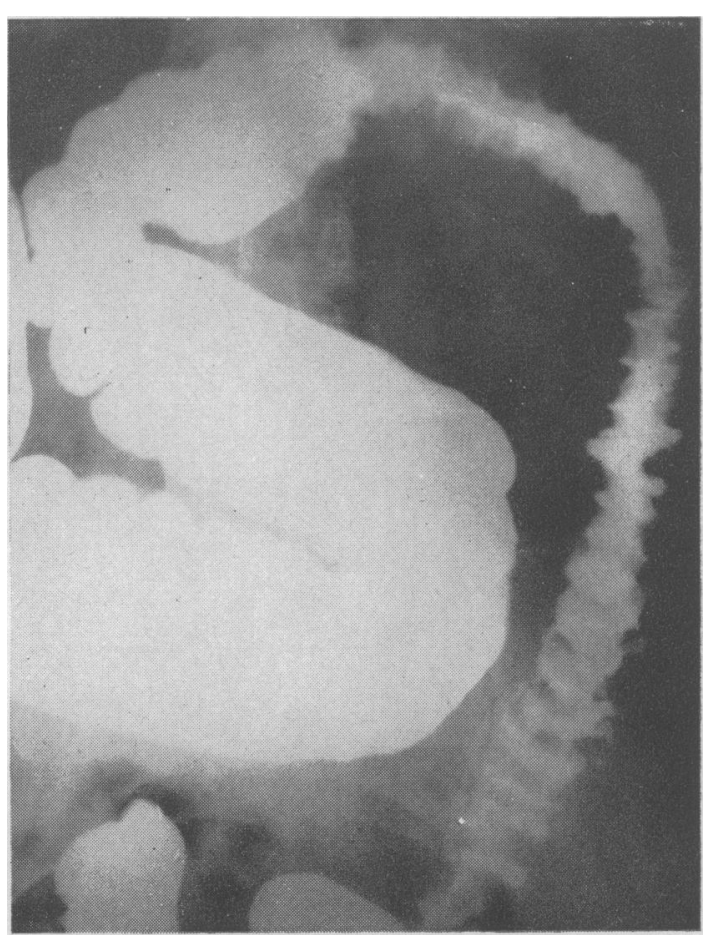

FIG. 5 .
FIG. 4.

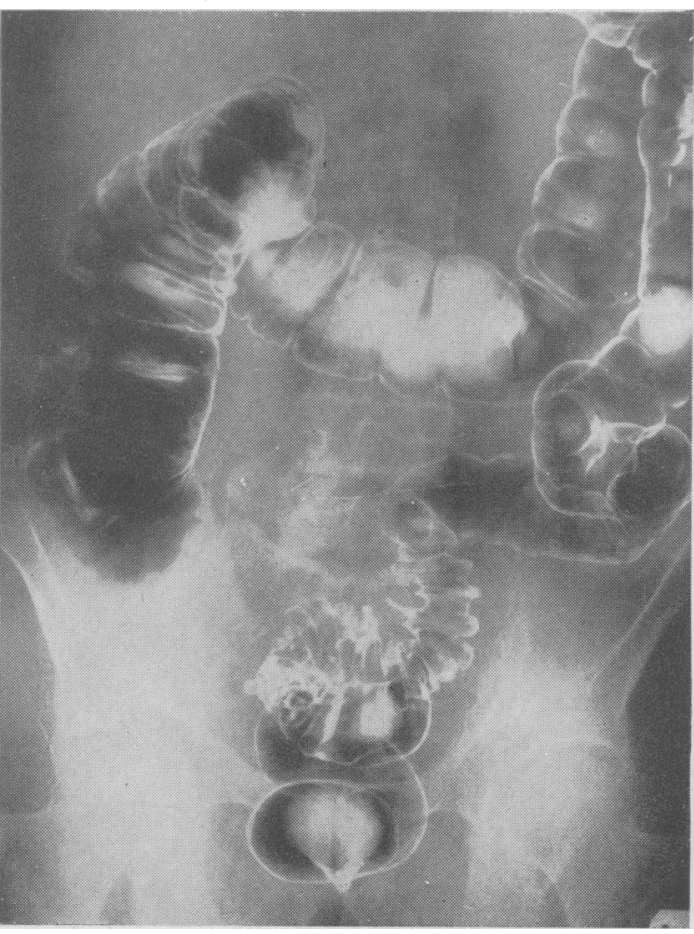

FIG. 4. Case 2: double contrast barium enema. The sigmoid region shows the characteristically asymmetrical fold pattern and several diverticula of diverticular disease. But on the medial aspect of the proximal curve (opposite the midpoint of the sacral promontory) is a deep spike accompanied by a shorter one. Such spiking might be due to non-specific diverticulitis, but in this case it is the spiking of Crohn's disease.

FIG. 5. Case 3: the sigmoid colon has an abnormal asymmetrical fold pattern and a few diverticula. The adjacent descending colon and the splenic flexure show an even more irregular fold pattern with well-marked fissuring, best seen in the splenic flexure.

This is a good example of the signs of diverticular disease being exceeded partly in extent, since gross fold changes are usually confined to the sigmoid, and partly in degree, since even in the sigmoid the irregular irregularity of the lumen and the fissuring would suggest Crohn's disease. 
volved by an inflammatory process causing marked thickening of the bowel wall with oedema of the mesentery. Subtotal colectomy was performed. Pathological examination of the resected specimen showed diffuse Crohn's disease of the colon with muscular hypertrophy and diverticulosis of the sigmoid (Figs. 6 and 7).

The patient has been seen at the follow-up clinic for five years and has had no further symptoms.

Case 4 A man of 61 , a lifelong sufferer from constipation, complained of anal discharge since the rupture of an abscess six months previously. A posterior anal fistula was present, and, on sigmoidoscopy to $18 \mathrm{~cm}$., blood was observed coming from above. A barium enema

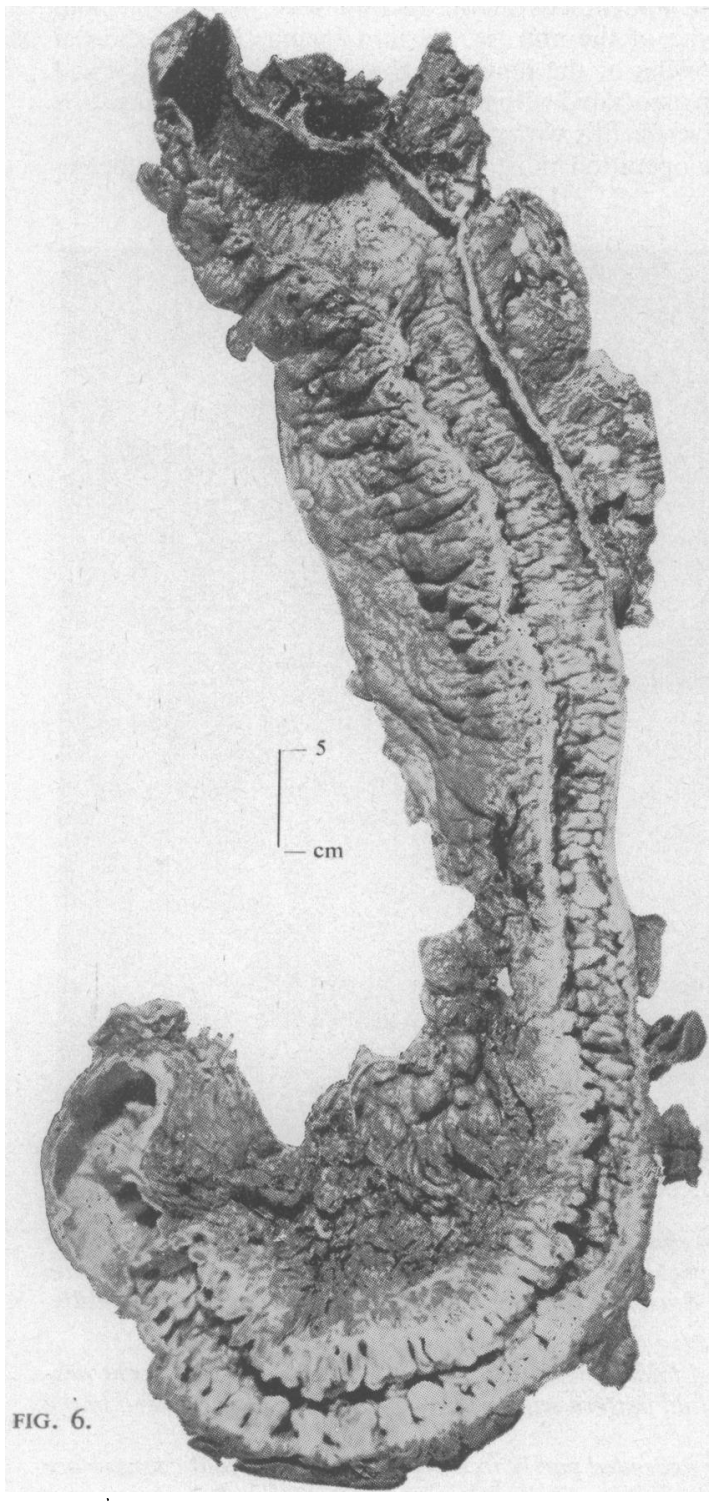

suggested severe diverticulitis of the sigmoid colon and a possible growth in the caecum. At laparotomy, the only abnormality found was gross thickening of the sigmoid colon attributed to diverticulitis. Resection of the diseased bowel with anastomosis was performed. Subsequently the anal fistula was laid open and healed normally. Examination of the resected specimen (Fig. 8) showed a few diverticula and, separate from the diverticula, chronic intramural inflammation, with hyperplasia of lymphoid tissue and many non-caseating giant cell systems. One lymph gland contained sarcoid foci. The track from the anal fistula also contained giant cell systems. The patient is well five years after operation.

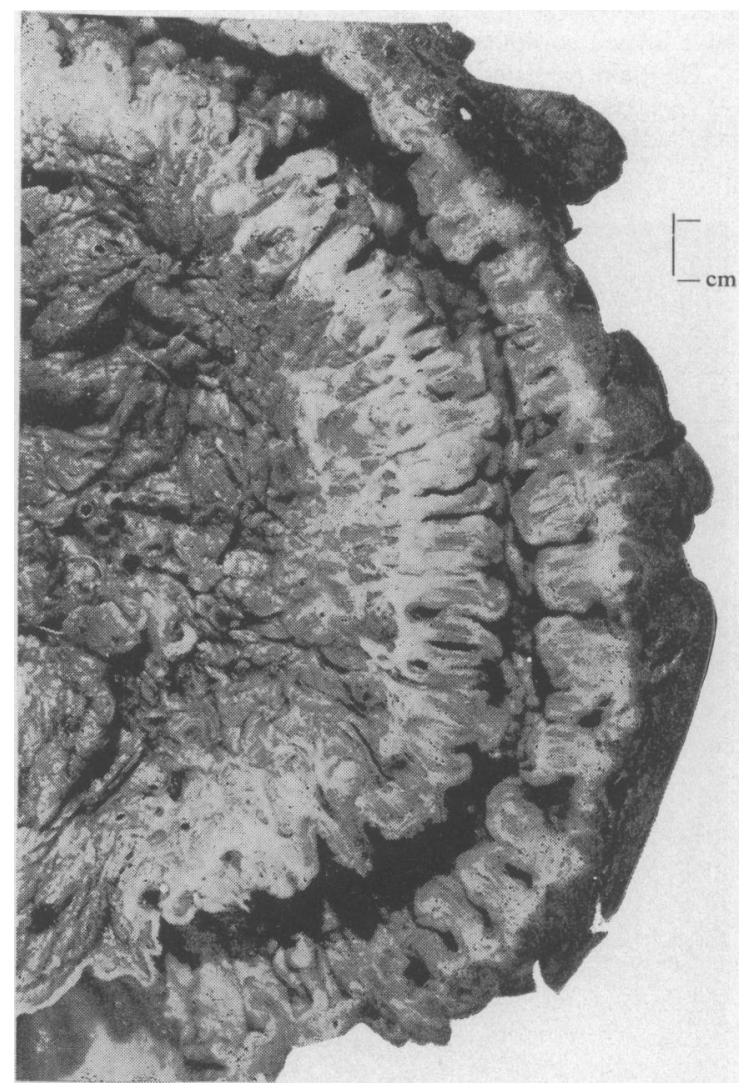

FIG. 7.

FIG. 6. Case 3: surgical specimen of left colon showing diffuse cobblestone appearance of the mucous membrane. In addition the sigmoid shows much muscular thickening and corrugation of the bowel wall typical of diverticular disease.

FIG. 7. Case 3: close up of sigmoid colon from Figure 6. Diverticula can be distinguished from the knife-like fissures of Crohn's disease passing through the bowel wall. Some diverticula show ulceration of their mucosal lining and there is extensive transmural inflammation extending into the pericolic fat with fibrosis. 


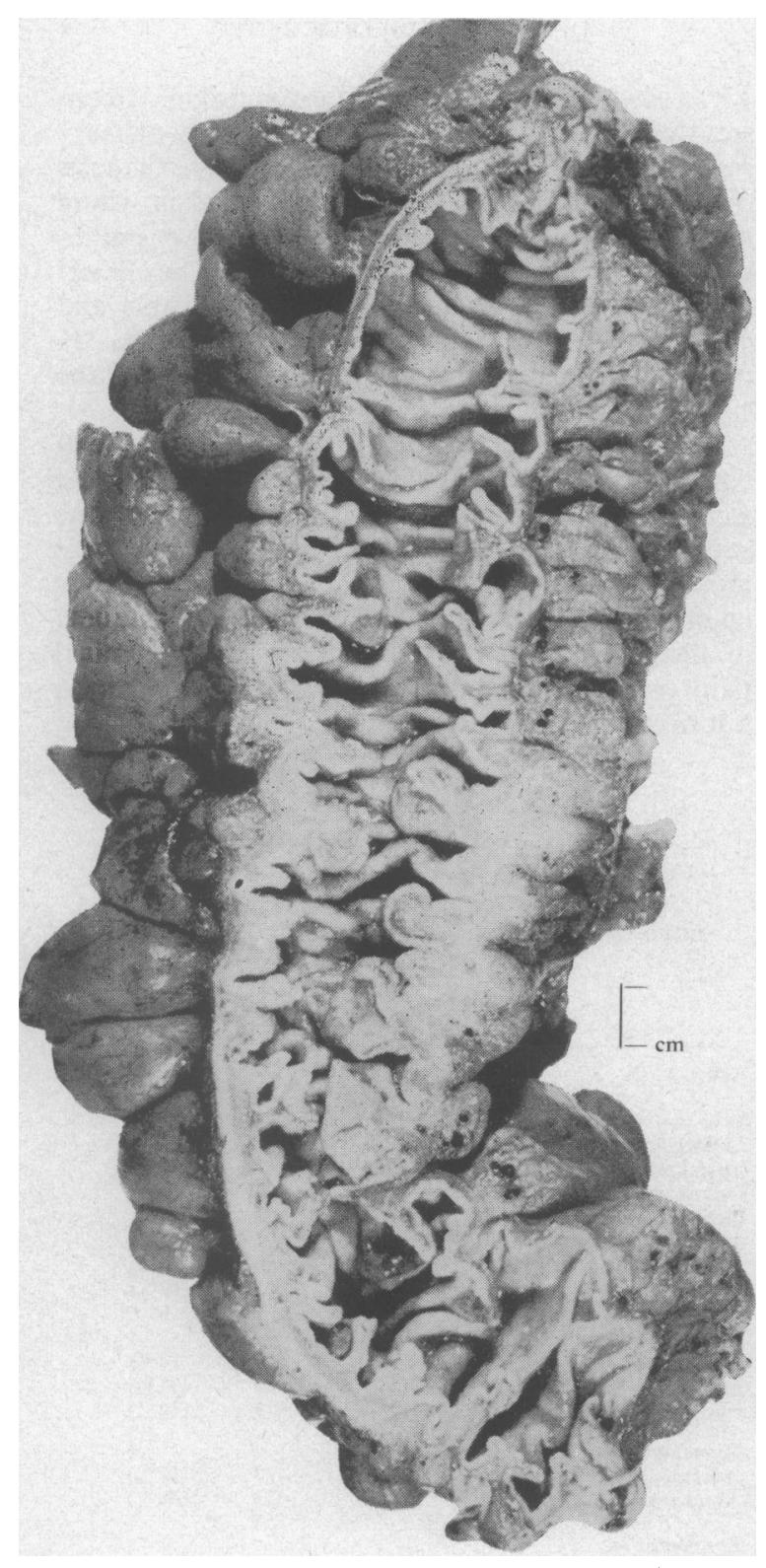

FIG. 8. Case 4: surgical specimen of sigmoid colon showing muscular thickening and corrugation of the bowel wall with mucosal redundancy and diverticulosis. The mucous membrane is thick and granular and in the central part of the specimen there is intramural and extramural fibrosis. Microscopic examination reveals features of Crohn's disease as well as diverticular disease.
GROUP II: DIAGNOSIS MADE ON A BIOPSY SPECIMEN In 12 patients the diagnosis of Crohn's disease was established by rectal biopsy or histological examination of tissue from anal lesions. In this group diverticula of the sigmoid were demonstrated radiologically but no resected specimen was available for examination. The initial clinical diagnosis was Crohn's disease in seven patients, diverticulitis in two, ulcerative colitis in two, and tuberculous proctitis in one. Crohn's disease, in addition to the diverticula, was suggested radiologically in nine patients. As in group I, the majority of these patients presented with diarrhoea (9), rectal bleeding (10), anal lesions (11), and an abnormal sigmoidoscopic appearance (12).

Case 5 is discussed in some detail as being a typical example of this second group.

Case 5 A male of 63 complained of anorectal discomfort for five months, and of passing frequent stools mixed with blood and mucus. On examination, several chronic anal fissures and a fistula were present and sigmoidoscopy revealed a granular inflamed mucosa with some blood and mucopurulent exudate in the lower rectum. Many sigmoid diverticula were seen radiologically (Fig. 9) as well as some granularity of the rectum with widening of the recto-sacral space. A clinical diagnosis of Crohn's disease was supported by a rectal biopsy which showed a chronic granulomatous infiltration with many sarcoid foci. After the fistula had been laid open the patient developed an exacerbation of symptoms with fever, abdominal pain, and distension. As it was difficult to be sure if these symptoms were due to the Crohn's disease or an attack of diverticulitis, he was treated at first with antibiotics but without effect. An immediate response followed the administration of prednisone ( $40 \mathrm{~g}$ daily), the dose of which was gradually reduced until the drug was stopped after nine weeks. He is well six months later, passing two or three formed stools daily, the anal lesions having healed five weeks after commencing treatment.

This case illustrates that there may be very real difficulty in deciding whether symptoms are due to diverticulitis or Crohn's disease. In this patient antibiotics were ineffective but corticosteroid therapy was successful.

GROUP III: CROHN'S DISEASE SIMULATING DIVERTICULITIS A further two patients were diagnosed as diverticulitis but pathological examination of the resected specimens showed Crohn's disease, predominantly of the sigmoid colon, without diverticula.

Case 6 A 59-year-old woman was transferred to this hospital four months after the onset of anorexia, weight loss of 3 stone, constipation, and the passage of blood and mucus on defaecation. At another hospital a hard, 


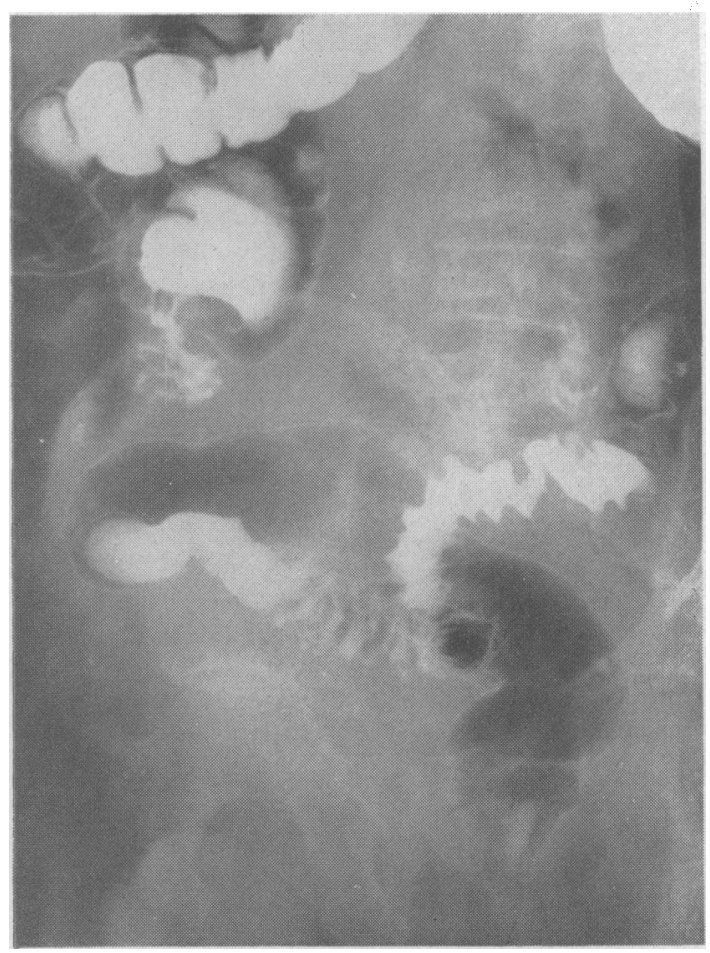

FIG. 9. Case 5: double contrast barium enema. The proximal sigmoid has an asymmetrical fold pattern (of a moderately regular type) and diverticula. The distal sigmoid has a diminished fold pattern; the rectum is irregular in outline and the original film showed fine granularity of the rectal mucosa.

fixed pelvic mass had been felt, and an oedematous haemorrhagic mucosa seen on sigmoidoscopy. Laparotomy revealed an inflammatory mass in the lower third of the sigmoid colon with thickening of the proximal two-thirds. A diagnosis of diverticulitis was made and a loop colostomy performed. Rectal biopsies were reported as showing active chronic inflammatory changes with partial ulceration of the mucosa.

After transfer to St. Mark's Hospital, no anal lesions were found and sigmoidoscopy showed an oedematous and granular mucosa with blood and pus coming from higher in the bowel. The diagnosis was thought to lie between Crohn's disease and diverticulitis. Barium enema and barium introduced via the distal loop of the colostomy (Figs. 10 and 11) showed diverticula in the descending colon together with narrowing and a spiky appearance reminiscent of previous cases in which Crohn's disease and diverticula were associated. A rectal biopsy was highly suggestive of Crohn's disease. Sigmoid resection was performed and histological examination confirmed the presence of Crohn's disease of the sigmoid but despite repeated examination no diverticula could be demonstrated macroscopically or microscopically.

\section{THE DIFFERENTIAL DIAGNOSIS BETWEEN CROHN'S DISEASE AND DIVERTICULITIS}

1 CLINICAL The records were examined of a consecutive series of 50 patients who had a primary resection at St. Mark's Hospital for diverticulosis or diverticulitis of the sigmoid colon and in whom no other lesion was found, either clinically or pathologically, to account for their symptoms. The clinical features in this series are set out in the Table and compared with the clinical features of the 26 patients with Crohn's disease and diverticula of the sigmoid colon.

It will be seen from the Table that the age incidence was about the same whether or not diverticula were associated with Crohn's disease. However, the sex incidence was reversed: diverticula alone were more common in men and combined disease was commoner in women. Bleeding was more frequent in those with combined disease while abdominal pain was commoner in those with diverticula but no Crohn's disease.

\section{TABLE}

CLINICAL FEATURES IN 50 PATIENTS OPERATED UPON FOR DIVERTICULOSIS OR ITS COMPLICATIONS COMPARED WITH THOSE IN 26 PATIENTS WTTH BOTH CROHN'S DISEASE AND COLONIC DIVERTICULA

$\begin{array}{llll}\begin{array}{l}\text { Diverticula without } \\ \text { Crohn's Disease } \\ \text { (50 patients) }\end{array} & \begin{array}{l}\text { Diverticula with } \\ \text { Crohn's Disease } \\ \text { (26 patients) }\end{array} \\ \left.\begin{array}{llll}\text { Range } & 39 \text { to } 86 \text { years } & \text { Range } & 48 \text { to } 80 \text { years } \\ \text { Mean } & 57.6 \text { years } & \text { Mean } & 62 \text { years } \\ 33 \mathrm{M} & 17 \mathrm{~F} & 11 \mathrm{M} & 15 \mathrm{~F}\end{array}\right) \\ 16 & (32 \%) & 2 & (8 \%) \\ 20 & (40 \%) & 16 & (62 \%) \\ 18 & (36 \%) & 20 & (77 \%) \\ 15 & (30 \%) & 7 & (27 \%) \\ 41 & (82 \%) & 5 & (19 \%) \\ 16 & (32 \%) & 3 & (12 \%) \\ & & & (0 \%) \\ 10 & (2 \%) & 0 & (85 \%) \\ 0 & (0 \%) & 22 & (12 \%) \\ 0 & (0 \%) & 3 & (12 \%) \\ 39 & (78 \%) & 3 & (88 \%) \\ 11 & (22 \%) & 23 & \end{array}$

Sigmoidoscop

Abnormal

Postoperative

Slow wound healing

Anal or recto-vaginal

fistulae

Anal fissure

Breakdown

anastomosis 


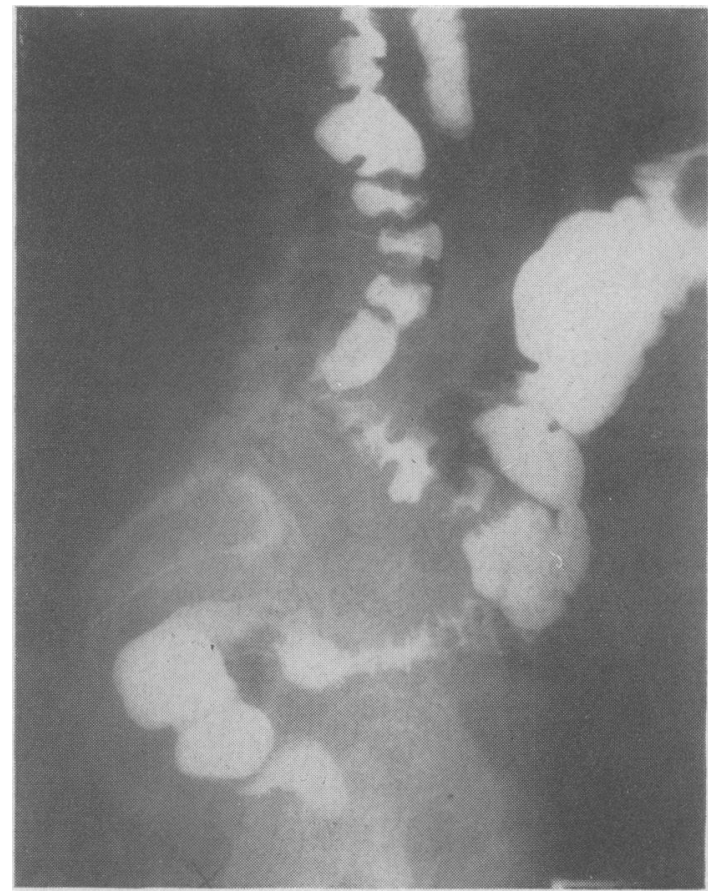

FIG. 10.

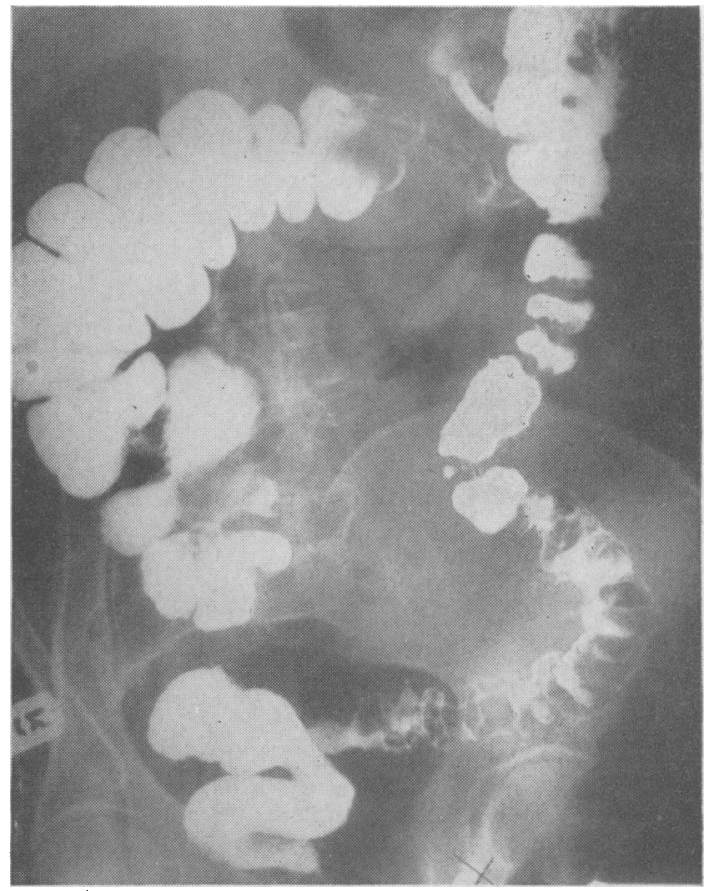

FIG. 11.

FIG. 10. Case 6: the proximal sigmoid shows an asymmetrical fold pattern suggestive of diverticular disease. The relatively long looped middle segment fills out fairly well, but the distal sigmoid is irregularly narrowed and fissured. The appearances in the distal segment are due to Crohn's disease; no diverticula were seen in the specimen.

FIG. 11. Case 6: enema via distal loop of colostomy. The sigmoid shows diverticula and asymmetrical folding, but there is also some fine transverse fissuring. It is hardly possible to make a radiological diagnosis of Crohn's disease here.

The incidence of anal lesions in the two groups was strikingly different. In patients with combined disease, 22 of 26 had anal lesions characteristic of Crohn's disease (Lockhart-Mummery and Morson, 1964) and two others had recto-vaginal fistulae. In contrast no anal lesions were present in the $\mathbf{5 0}$ patients with diverticula alone.

Another marked difference between the two groups was the high incidence of sigmoidoscopic abnormalities among those with combined disease. The rectal mucosa was abnormal in 19 (friable, granular, nodular, or ulcerated) of the 26 such patients; three others showed blood or mucopus in the lumen without mucosal change, and stenosis of the anal canal was present in another. In contrast the sigmoidoscopic appearances were abnormal in only 11 of the 50 patients with diverticula alone; seven showed mucosal changes and four had a normal mucosa but with blood or pus present in the lumen.

The distinction between the two groups is shown to be of practical importance in treatment by the high incidence of postoperative complications after resection. Among those with combined disease seven of the 14 patients developed postoperative complications: in three the colonic anastomosis broke down to give an external fistula; three developed recto-vaginal fistula; the wound healed slowly in two, and chronic anal fissures and fistulae also occurred. The only such complication among 50 patients with diverticula alone was an anal fistula.

2 RADIOLOGICAL Uncomplicated diverticular disease can be recognized by a deep, asymmetrical fold pattern almost always accompanied by diverticula. Diverticulitis may lead to abscess formation, an internal fistula, or fibrosis (Young, 1965). It may be impossible to differentiate the changes of Crohn's and diverticular disease when both affect the same segment of colon and, as already shown, Crohn's disease may be mistaken for diverticular disease. 
The following radiological features have been found helpful in diagnosis.

An internal or external fistula should suggest the possibility of Crohn's disease. Very deep, narrow spurs of barium (Fig. 4) are unusual in diverticular disease. When the radiologist sees narrowing of the sigmoid with barium projecting in long, thin streaks at right angles to the axis of the bowel, he may be in doubt as to whether this represents the close-packed folds of diverticular disease or the fissuring of Crohn's disease. If the projections of barium show a regular asymmetry, and especially if they terminate in a sacculation, he is probably looking at diverticular disease. But if the projections are spiky, blind and haphazardly distributed, he should at least consider the possibility of Crohn's disease (Figs. $9,10,11)$.

It is unusual for diverticular disease to involve more than the sigmoid colon and an inflammatory lesion of unusual extent suggests additional pathology (Fig. 5). Any abnormality of the large bowel at a distance from the diverticula, for example mucosal ulceration, should also suggest an associated condition. Similarly rectal abnormalities such as a granular mucosal pattern, narrowing with an increased recto-sacral distance, or frank ulceration are not features of diverticular disease (Fig. 9). For this reason, it is essential that the barium enema films should include films of the rectum and always include one lateral view.

3 pathological The diagnosis of Crohn's disease and diverticulitis was made by examination of resected specimens of diseased bowel in 14 of the 26 patients included in this study. In all these cases the diverticular disease mainly affected the sigmoid colon, but the Crohn's disease often involved other parts of the large intestine or anus as well. The criteria for the pathological diagnosis of diverticular disease (Morson, 1963) and of Crohn's disease (Hadfield, 1939; Lockhart-Mummery and Morson, $1960,1964)$ have been described elsewhere.

Macroscopic appearances In specimens of combined Crohn's disease and diverticulitis the characteristic features of diverticular disease are easily recognizable by macroscopic examination. It is the presence of diverticula with the muscular thickening and corrugation of the bowel wall which are important. Indeed, the first impression given by examination of these specimens was obvious diverticular disease with extensive pericolic inflammation. Closer inspection, however, revealed additional features not usually associated with diverticulitis.

The mucous membrane of the sigmoid colon in diverticulitis usually appears normal except for the redundancy consequent upon the muscular shorten- ing. Redundancy was a feature of some of the specimens of combined Crohn's disease and diverticulitis reported here but in addition all cases showed mucosal inflammation. This took the form of, a granular appearance with oedema and mucosal thickening, often with patches of full-thickness ulceration of the mucous membrane. This was variable in extent and was associated in two cases with inflammatory polyp formation due to undermining of mucosa adjacent to areas of ulceration. The mucosal inflammation was not particularly associated with the orifices of diverticula, although in one case (Fig. 2) this was rather prominent. The cut surface of the bowel wall showed evidence of transmural and extramural inflammation, whereas in uncomplicated diverticulitis the inflammatory component of the pathology is exclusively pericolic in position. The fissuring so characteristic of Crohn's disease was present (Figs. 3 and 7) in most of these cases, but is best observed at a microscopic level of observation.

Microscopic appearances The most reliable criteria for the diagnosis of Crohn's disease are the presence of a granulomatous or sarcoid type of tissue response with a transmural type of inflammation and fissuring of the bowel wall. In the specimens of Crohn's disease and diverticulitis reported here the former diagnosis depended upon the presence of a sarcoid type of tissue response which was present in the bowel wall in all cases and in the regional lymphatic glands in 11 out of the 14 surgical specimens. These sarcoid foci were found in the mucosa, submucosa, and muscle layers as well as in the pericolic tissues. Fissuring could be found in most cases but was especially prominent in three. It could be distinguished, even macroscopically in some specimens, from the breaching of the bowel wall by diverticula. Transmural inflammation with widening and oedema of the submucosa, together with many foci of lymphoid tissue, was also characteristic. These microscopic features were present in addition to the diverticula and muscular changes of diverticular disease.

The inflammation in diverticulitis is extramural. Foci of lymphoid tissue are commonly seen around chronic pericolic abscess cavities but do not involve the submucosal and deep muscular layers of the bowel wall. Intramural fissuring is not a feature of diverticulitis and a true sarcoid reaction is never seen in the bowel wall or regional lymph nodes. However, the distinction between a foreign-body giant cell reaction in the neighbourhood of pericolic abscesses in diverticulitis and a true sarcoid reaction may not be easy. In most of such cases, however, the giant cells are arranged around material obviously of faecal origin or else they are clearly foreign body 
rather than the Langhans type. Moreover, it must be emphasized that a giant cell reaction in diverticulitis is extramural around infected diverticula.

Diagnosis of Crohn's disease in biopsies of the rectum and anal lesions In 12 patients the diagnosis of Crohn's disease was made by rectal biopsy or biopsy of an anal lesion. The principal diagnostic feature in all cases was the presence of sarcoid granulomata as described in previous papers (Morson and Lockhart-Mummery, 1959; LockhartMummery and Morson, 1964).

\section{DISCUSSION}

Review of the literature on Crohn's disease of the colon and on diverticulosis has revealed few references to this problem of combined disease. LockhartMummery and Morson (1964) pointed out that the clinical distinction between Crohn's disease and diverticulosis of the sigmoid colon is not always easy, and Marganoff (1966) has indicated that it may be difficult to differentiate early localized lesions of Crohn's disease from carcinoma and diverticulitis.

The importance of diagnosing the presence of Crohn's disease is suggested by the fact that of the nine patients in this series operated on with a preoperative diagnosis of 'diverticulitis', six developed postoperative complications (breakdown of anastomosis with cutaneous fistula, two fistula-in-ano, and two slow wound healing). On the other hand, of the four patients diagnosed as having Crohn's disease and diverticula preoperatively, only one developed a postoperative complication (ano-rectal suppuration). If cutaneous fistula or ano-rectal c omplications develop after a resection for 'diverticulitis' Crohn's disease should be considered, and the resected specimen or any biopsy material should be re-examined with this possibility in mind.

The knowledge that Crohn's disease is present may also incline the clinician towards medical management, rather than sigmoid resection, in some cases. Sulphonamides appear to benefit some of these patients; in others topical or systemic corticosteroids may be most useful, whereas these drugs would not be used for the treatment of diverticulitis.

Whatever part of the gastrointestinal tract is involved only about half of all patients with Crohn's disease have a sarcoid type of tissue response. The precise incidence largely depends on how energetically the search for granulomas is pursued. In some cases these are sparsely distributed. Although granulomas were present in all the 26 cases reported here it is likely that Crohn's disease and diverticulitis may coexist in the sigmoid colon in the absence of granulomas. In such cases the pathological diagnosis would be difficult and the diagnosis of Crohn's disease would depend on other features, such as transmural inflammation and fissuring. This situation has not yet arisen in our experience, but it is possible that the diagnosis of 'diverticulitis' alone has been made in patients who also have unrecognized Crohn's disease in addition to diverticula. In such a situation the findings of a clinically characteristic anal lesion or proctitis in association with sigmoid diverticulitis would lead at least to a suspicion of Crohn's disease.

\section{SUMMARY}

Twenty-six patients in whom Crohn's disease occurred in a segment of bowel containing diverticula have been reviewed. In 14 of the patients the diagnosis was made by examination of a resected specimen; in 12 it was made by examination of biopsy material from the anus or rectal mucosa.

Twenty-four of the 26 patients had an anal lesion or recto-vaginal fistula and in 23 there were $a b$ normal findings on sigmoidoscopy. In only one patient did the anus and rectum appear normal, but rectal mucosal biopsy was abnormal. These features were more common than among 50 patients operated on for diverticulosis, or its complications, alone.

Radiologically, Crohn's disease in the presence of diverticula was suggested by unusual fissuring, a mucosal lesion involving the rectum or colon away from the area of diverticular disease, or a fistula.

Biopsy revealed non-caseating giant-cell granulomata in 10 of 11 biopsies of anal lesions and 12 of 13 biopsies from the rectal mucosa. Pathological examination of resected specimens from patients with combined disease showed a diffuse mucosal lesion, transmural inflammation with the presence of fissures and non-caseating giant-cell systems.

The distinction between Crohn's disease and diverticulitis may be important in treatment. Postoperative complications were more common after resection in patients with combined disease than after resection for diverticulosis or its complications alone. The knowledge that Crohn's disease is present may affect medical management, particularly regarding the use of corticosteroids.

We thank our colleagues at St. Mark's Hospital for their help in this study, and the St. Mark's Research Foundation and the Research Endowment Fund of the Hospital for financial support.

\section{REFERENCES}

Arfwidsson, S. (1964). Pathogenesis of multiple diverticula of the sigmoid colon in diverticular disease. Acta chir. scand., suppl. 342. 
Brooke, B. N. (1959). Granulomatous diseases of the intestine. Lancet, 2, 745-749.

Crohn, B. B., and Yarnis, H. (1958). Regional Ileitis, 2nd ed. Grune and Stratton, New York and London.

Dearlove, T. P. (1954). Diverticulitis and diverticulosis, with a report on a rare complication. Med. J. Aust., 1, 470-475.

Hadfield, G. (1939). The primary histological lesion of regional ileitis. Lancet, 2, 773-775.

Lindner, A. E., Marshak, R. H., Wolf, B. S., and Janowitz, H. D. (1963). Granulomatous colitis: a clinical study. New Engl. J. Med., 269, 379-385.

Lockhart-Mummery, H. E., and Morson, B. C. (1960). Crohn's disease (regional enteritis) of the large intestine and its distinction from ulcerative colitis. Gut, 1, 87-105.

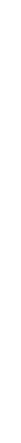

$-\frac{(1904)}{493-509 .}$
Marganoff, H. colitis. Dis. Colon Rect., 9, 49-57.

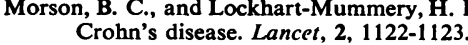

Morson, B. C. (1963). The muscle abnormality in diverticular disease of the sigmoid colon. Brit. J. Radiol., 36, 385-392.

Pemberton, J. deJ., Black, B. M., and Maino, C. R. (1947). Progress in the surgical management of diverticulitis of the sigmoid colon. Surg. Gynec. Obstet., 85, 523-534.

Williams, I. (1963). Changing emphasis in diverticular disease of the colon. Brit. J. Radiol., 36, 393-406.

Young, A. C. (1965). In panel discussion on surgical management of diverticulitis. Dis. Colon Rect., 8, 167-169. 